\title{
Implementation of digital and analog modulation systems using FPGA
}

\author{
Raya. K Mohammed, Hamsa A. Abdullah
}

College of Information Engineering, Al-Nahrain University, Iraq

\begin{tabular}{l}
\hline \hline Article Info \\
\hline Article history: \\
Received Jul 9, 2019 \\
Revised Sep 12, 2019 \\
Accepted Sep 26, 2019 \\
\hline
\end{tabular}

\section{Keywords:}

Analog modulation Digital modulation FPGA

System generator Vivado

\begin{abstract}
FPGA (Field Programmable Gate Array) based implementations of digital and analog modulation techniques play a vital rule in the design of signal processing system. The performance and flexibility provided by reconfigurable computing speeds up the development process in signal processing implementations using FPGA. Different methods for digital and analog modulation are designed in this paper by usinSg System Generator tools \& Vivado. Then all designed systems are implemented successfully in an FPGA hardware via the NEXYS 4 DDR with ARTIX 7 XC7A100T. A comparison between five types of digital modulation techniques is discussed in terms of resources utilization in FPGA hardware. And also, the implementation of analog modulations in FPGA is contributed in this work. The hardware implementation shows that the number of slice LUTs in ASK modulation is $0.07 \%$ while in FSK modulation is $0.13 \%$ of the total number of slice LUTs. And also, the number of bounded IO that used for PSK modulation is $4.8 \%$ while in PM modulation is $61.4 \%$ of the total number bounded IO.
\end{abstract}

Copyright (C) 2020 Institute of Advanced Engineering and Science. All rights reserved.

\section{Corresponding Author:}

Raya K. Mohammed,

Department of Information and Communications,

College of Information Engineering, Al-Nahrain University,

Baghdad, Iraq.

Email:rayakhahtan@coie-nahrain.edu.iq

\section{INTRODUCTION}

Understanding and processing information has become increasingly needed due to the increasing use of computers. And because of these, the usage of signal processing has increased. A digital signal processor (DSP) is a particular form of microprocessor, while an FPGA is an excellent alternative design platform in the implementation of digital systems due to their ability to quickly process parallel hardware designs with minimal cost. In DSP system's implementations, FPGAs became a basic part particularly in fields such as digital communications [1-4].

Communication is the process of transfer information. It means that the transmitter sends the encoded symbols through the communication channel, and the receiver decoded the information to recover the original data $[5,6]$. The modulation method is used in the communication process that increases multiplexing, the range of communication, and improves the quality of reception [7]. It is defined as the method of covering the information contents of a baseband signal on a carrier signal [8].

In [9], Quarter Phase Shift Keying (QPSK) modulator is designed using ISE system generator with Hardware Co-simulation tools. Then the designed system is converted to VHDL (Very High-Speed Integrated Circuit Hardware Descriptive Language). ISE Simulation software is used to synthesize the converted VHDL code and implement it in an FPGA. In this work the proposed QPSK modulator is implemented in an FPGA at low power. In [10], Digital Modulation Technique is implemented in FPGA for High Range Resolution (HRR). In this work, designing Binary Phase Shift Keying (BPSK) modulation is 
considered with different methods. In [11], BASK, BFSK, DPSK and BPSK are designed using ISE system generator and implemented in FPGA using Nexys 2 with Spartan 3E board.

However, the above proposed works have traditional implementation for some digital modulation and they do not consider all type of digital modulation techniques. Furthermore, they do not consider the use of analog modulation techniques for FPGA hardware implementation. So, this work is an effort to consider all these issues to provide a digital and analog modulation. In this paper, the hardware implementation of digital and analog modulation is introduced. The implementation process is based on using a hardware programmable device (FPGA) with low cost, high speed, flexible and reconfigurable experimental setup. This paper is prepared as follows. Section 2 includes description of digital modulation techniques. Section 3 includes analog modulation techniques. Section 4 and 5 include FPGA implementation of digital and analog modulation respectively. Section 6 includes experimentalism results. Finally, Section 7 gives the conclusions of the paper.

\section{METHODOLOGY}

There are two main types of modulation techniques, digital and analog. In this work, five kinds of digital modulation and three kinds of analog modulation methods are considered.

\subsection{Digital Modulation Techniques}

Digital modulation indicates the method of transmitting a digital signal with low-frequency over a carrier signal with higher-frequency [12]. In radio communications system, modulation is supported by varying the characteristic of carrier sinusoidal signal (amplitude, phase, frequency, or any combination of them). At the transmitter side, the modulator imposes the physical change to the carrier. And at the receiver side, a demodulator recovers the information [6]. The most used digital modulation methods are:

\subsubsection{Binary Amplitude Shift Keying (BASK)}

In a BASK modulation, the carrier signal's amplitude is altered in consistent with the message bit's values without changing the frequency and phase. The BASK modulated signal is represented by the carrier signal when the message data is 1 , otherwise, it is represented by 0 . In modulation process, the modulated signal is created by multiplying the bits data with a carrier signal [13, 14].

$$
S(t)= \begin{cases}A_{c} \sin \left(2 \pi f_{c} t\right) ; & \text { if message data }=1 \\ 0 ; & \text { if message data }=0\end{cases}
$$

\subsubsection{Binary Frequency Shift Keying (BFSK)}

In a BFSK modulation, the carrier signal's frequency is altered in consistent with the message bit's values without changing the amplitude and phase $[15,16]$. In BFSK, two carrier signals are used in modulation. The modulated signal is represented by the first carrier signal when the message data is 1 , while it is represented by the second carrier signal when the message data is 0 [17].

$$
S(t)= \begin{cases}A_{c} \sin \left(2 \pi f_{1} c t\right) ; & \text { if message data }=1 \\ A_{c} \sin \left(2 \pi f_{2} c t\right) ; & \text { if message data }=0\end{cases}
$$

Where $f_{s} \ll f_{1} \& f_{2}$.

\subsubsection{Binary Phase Shift Keying (BPSK)}

In a BPSK modulation, the sinusoidal carrier signal's phase is altered in consistent with the message bit's values without changing frequency and amplitude [15, 18]. In BPSK, the modulated signal is represented by the positive value of the carrier signal when the message data is 1 , while it represented by the negative value of the carrier signal when the message data is $0[19,20]$.

$$
S(t)= \begin{cases}A_{c} \sin \left(2 \pi f_{c} t\right) ; & \text { if message data }=1 \\ -A_{c} \sin \left(2 \pi f_{c} t\right) ; & \text { if message data }=0\end{cases}
$$

\subsubsection{Quarter Phase Shift Keying (QPSK)}

QPSK modulation is based on phase modulation. Two bits are used to represent each symbol, I and $Q$ channels are used to modulate these bits. Sine and cosine waves are used as carrier signals for $I$ and $Q$ channels respectively. There are four cases in QPSK modulation: (00, 01, 10 and 11) [21]. In each case, different phases are used to create QPSK and these phases are $0^{\circ}, 90^{\circ}, 270^{\circ}$ and $360^{\circ}$. The symbol period is 
double the bit period $T_{s}=2 T_{b}$ [8]. As compared with traditional digital modulation methods (BFSK BPSK BASK), QPSK is considered as fast modulation method [22,9].

\subsubsection{Differential Phase Shift Keying (DPSK)}

DPSK is a mutual method of phase modulation which transfers binary data by varying the phase of the carrier signal. As well as in BPSK and QPSK, there is no insurance which signal is passed according to its phase if the constellation is rotated by some influence in the communications channel. This issue can be solved by using the data to alteration instead of set the phase. In DPSK, the modulated signal's phase is shifted corresponding to the earlier signal element. DPSK doesn't required a synchronous carrier at the receiver. The modulated signal is represented by the negative value of the carrier signal when the message data is 0 , while it represented by the shifted phase of the preceding carrier signal when the message data is 1 . For that reason, in the receiver, the preceding received bits are used to distinguish the current bit [11].

$$
S(t)= \begin{cases}A_{c} \cos \left(2 \pi f_{c} t+\theta(t)+\theta\right) ; & \theta \text { is } \pi \text { if message data }=1 \\ A_{c} \cos \left(2 \pi f_{c} t+\theta\right) ; & \theta \text { is } 0 \text { if message data }=0\end{cases}
$$

where $\theta(t)$ is phase information and $\theta$ is the phase shift presented by the channel.

\subsection{Analog Modulation Techniques}

Analog modulation indicates the method of transmitting an analog baseband signal with lowfrequency over a carrier signal with higher-frequency. In this type of modulation, an analog signal is used to represent the baseband. The main three types of analog modulation are: amplitude modulation, frequency modulation, and phase modulation [23].

\subsubsection{Amplitude Modulation Technique (AM)}

In AM, the carrier signal's amplitude is changed in consistent with the message signal's amplitude. Suppose that the message signal is $m(t)$ and the carrier signal is $(t)=A \cos \left(w_{c} t\right)$, then the $A M$ signal $Y(t)$ is represent by $[24,25]$ :

$$
Y(t)=[A+m(t)] \cos \left(w_{c} t\right)
$$

\subsubsection{Frequency Modulation Technique (FM)}

In FM, the carrier signal's frequency is changed in consistent with the message signal's amplitude. Suppose that the message signal is $m(t)$ and the carrier signal is $c(t)=A \cos \left(w_{c} t\right)$, then the $F M$ signal $Y(t)$ is represent by:

$$
\mathrm{Y}(\mathrm{t})=\mathrm{A} \cos \left(\mathrm{w}_{\mathrm{c}} \mathrm{t}+\mathrm{k}_{\mathrm{f}} \int \mathrm{m}(\alpha)\right) \mathrm{d} \alpha
$$

\subsubsection{Phase Modulation Technique (PM)}

In PM, the carrier signal's phase is changed in consistent with the message signal's amplitude. Suppose that the message signal is $m(t)$ and the carrier signal is $c(t)=A \cos \left(w_{c} t\right)$ then $P M$ signal $Y(t)$ is represent by:

$$
\mathrm{Y}(\mathrm{t})=\mathrm{A} \cos \left(\mathrm{w}_{\mathrm{c}} \mathrm{t}+\mathrm{k}_{\mathrm{p}} \mathrm{m}(\mathrm{t})\right)
$$

\section{RESEARCH METHOD}

In this paper, different types of digital and analog modulation techniques are considered. All these techniques are designed and simulated in Xilinx System Generator which is a configurable tool for FPGA implementations. The designed systems are converted to VHDL codes that are used as inputs to Vivado Design Suite. Then the bitstreams for all types of modulations are generated successfully and downloaded to FPGA hardware.

\subsection{Digital Modulation's Implementation in System Generator}

In this paper, five techniques for digital modulation are designed to offer a structure that can be used in a particular application in consistent with the requirements and the environment of that application. The most common used approaches for FPGA's implementation of modulation techniques is system generator environment. The message signals in all systems are generated externally to be connected directly to the input ports of the FPGA hardware. 


\subsubsection{BASK Implementation}

Figure 1 shows the BASK implementation in System Generator. In Figure 1(a) the BASK modulation system is considered. The message signal is externally generated, while the carrier signal is internally generated. Figure 1(b) displays the carrier signal (discrete sine wave), in addition to the message signal, and the ASK modulated signal holding together with the message signal. The used system generator blocks are: discrete sine wave, gateway in, gateway out, mux, pulse generator, and scope blocks. Some of the gateway out blocks are disconnected from the hardware connection to reduce the resources utilized in FPGA.

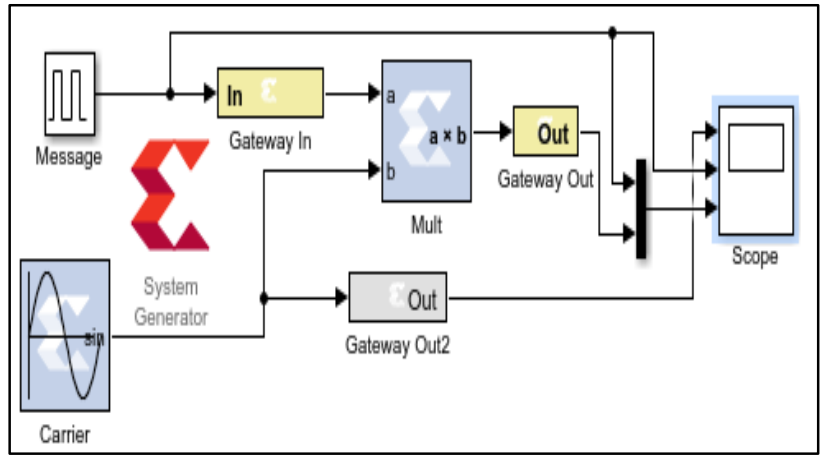

(a)

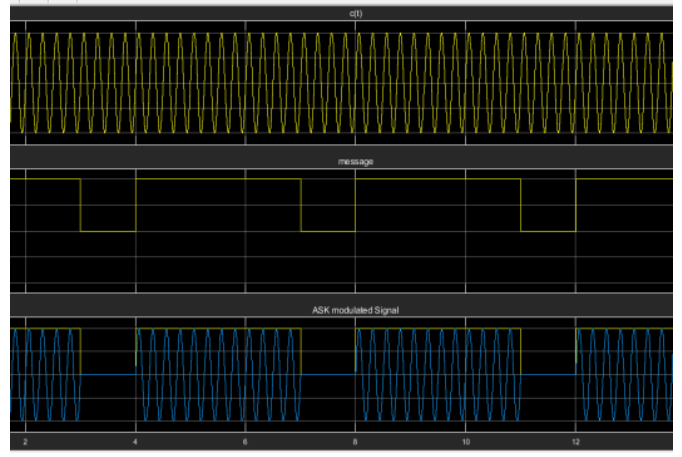

(b)

Figure 1. BASK implementation in system generator. (a) BASK Modulation system,

(b) The BASK input \& output signals

\subsubsection{BFSK Implementation}

Figure 2 shows the BFSK implementation in System Generator. In Figure 2(a), the BFSK modulation system is considered. the message signal is externally generated, while the carrier signals are internally generated. Figure 2(b) displays the carrier signals (discrete sine waves), in addition to the message signal, and the FSK modulated signal holding together with the message signal. The used system generator blocks are: discrete sine wave, gateway in, gateway out, mux, convert, pulse generator and scope blocks.

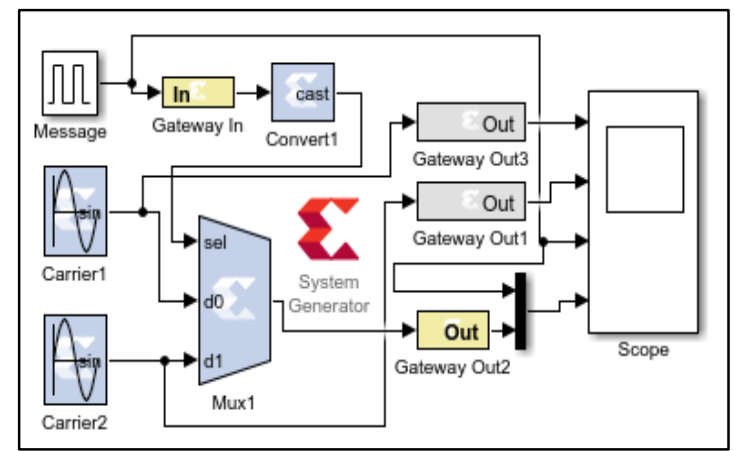

(a)

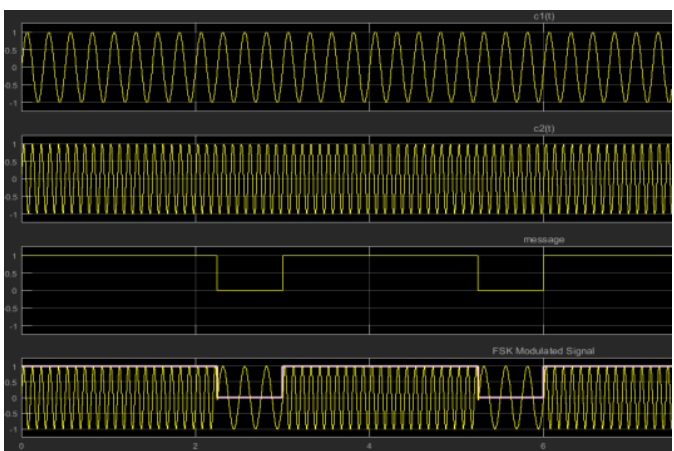

(b)

Figure 2. BFSK implementation in system generator. (a)BFSK modulation system,

(b) The BFSK input \& output signals

\subsubsection{BPSK Implementation}

Figure 3 shows the BPSK implementation in System Generator. In Figure 3(a), BPSK modulation system is considered. One of the carrier signals is internally generated, while the second one is produced by multiplying the first one by (-1). The message signal is externally generated. Figure 3(b) displays the carrier signals (discrete sine waves), in addition to the message signal, and the PSK modulated signal. The used System Generator Blocks are: gateway in, gateway out, discrete sine waves, mux, convert, pulse generator, Cmult blocks and scopes. 


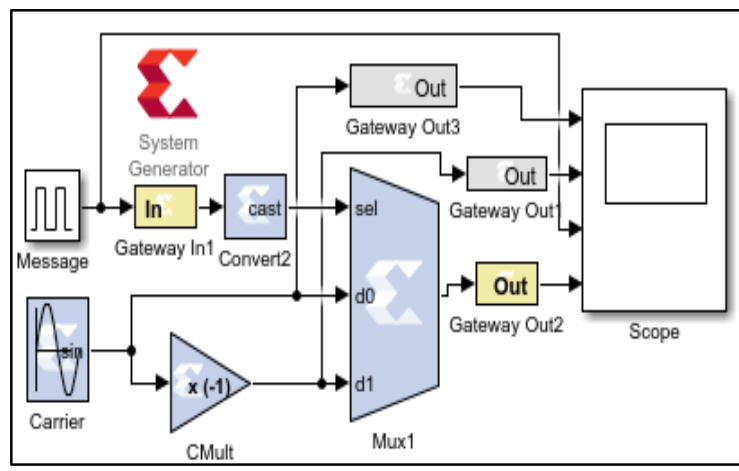

(a)

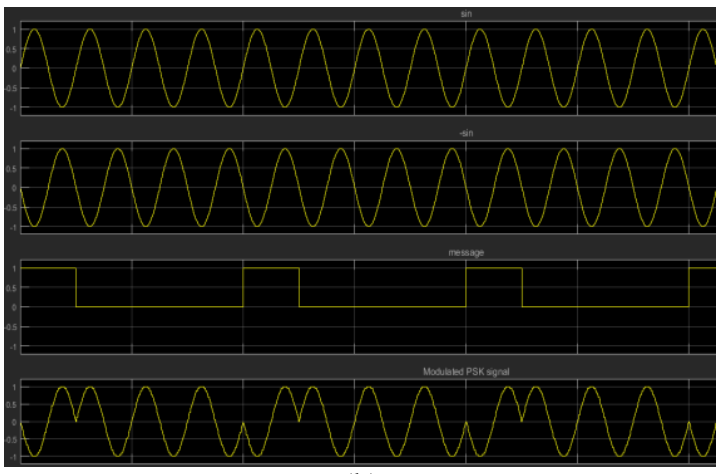

(b)

Figure 3. BPSK implementation in system generator. (a) BPSK modulation system,

(b) The BPSK input \& output signals

\subsubsection{DPSK Implementation}

Figure 4 shows the DPSK implementation in System Generator. In Figure 4(a), DPSK modulation system is considered. One of the carrier signals is internally generated, while the second one is produced by multiplying the first one by (-1). The message signal is externally generated. Figure 4(b) displays the message signal, differentially encoded signal, in addition to the DPSK modulated signal. The used system generator blocks are: the gateway in, the gateway out, sine wave (discrete), Cmult, mux, convert, logical, delay, and scopes blocks.

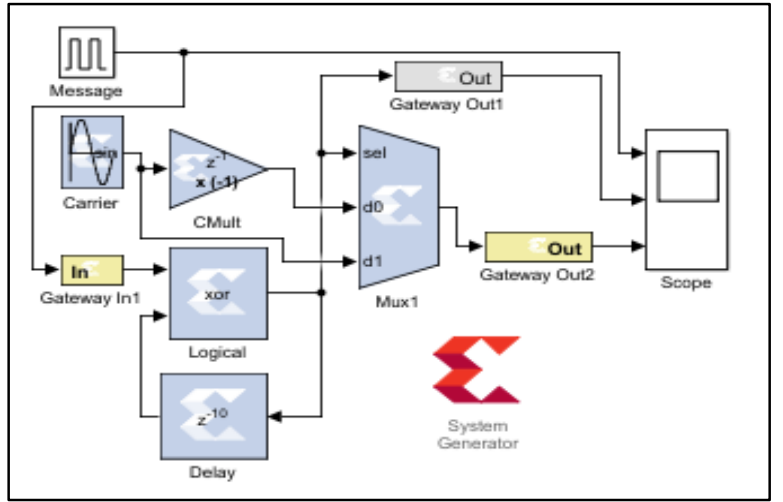

(a)

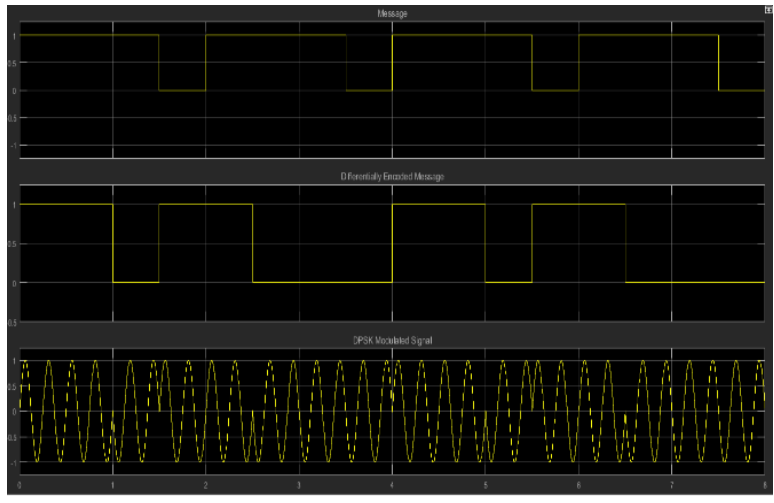

(b)

Figure 4. DPSK implementation in system generator. (a) DFSK modulation system,

(b) The DPSK input \& output signals

\subsubsection{QPSK Implementation}

Figure 5 shows the QPSK implementation in System Generator. In Figure 5(a), the QPSK modulation system is considered. The carrier signals are externally generated, while the message signal is internally generated by LFSR and TDD. Figure 5(b) illustrates the message signal, the output PSK signal of I channel and the output signal of Q channel, in addition to the QPSK modulated signal. The used system generator blocks are: discrete sine waves, gateway in, gateway out, TDD, LFSR, AddSub, Mux and scopes blocks. 


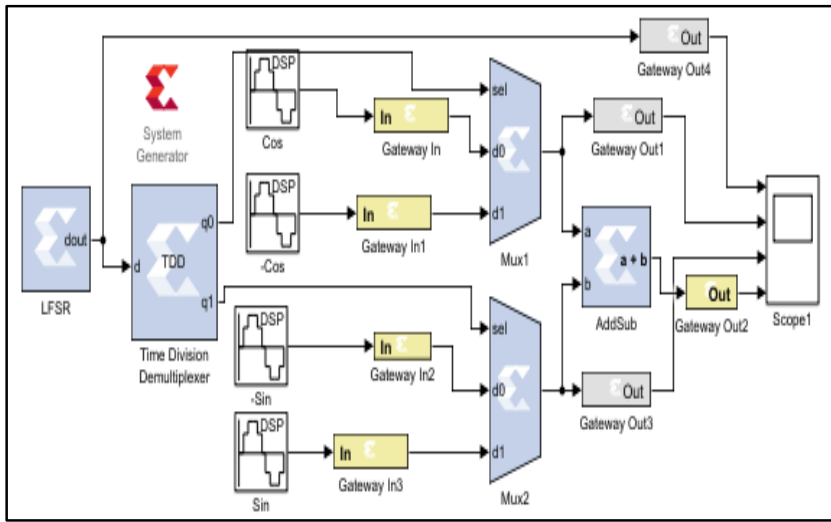

(a)

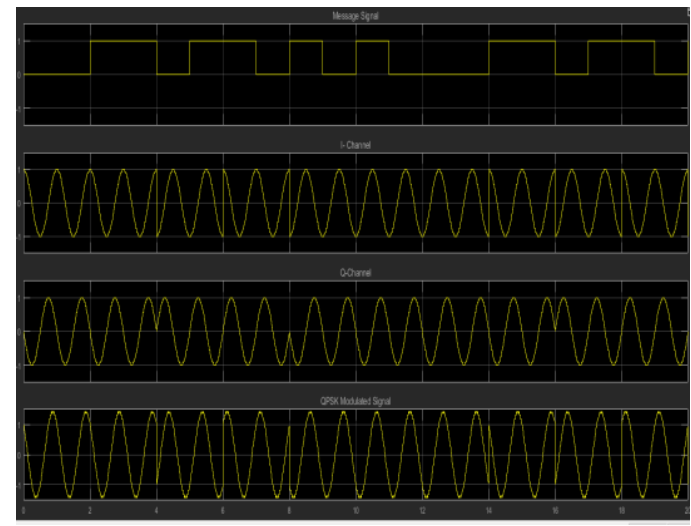

(b)

Figure 5. QPSK implementation in system generator. (a) QFSK input \& output signals,

(b) The QPSK input \& output signals

\subsection{Analog Modulation Implementation in System Generator}

In this paper, three main analog modulation techniques are designed to offer a structure that can be used in a particular application in consistent with the requirements and the environment of that application. The main contribution of this paper is the implementation of analog modulation in an FPGA using Vivado \& System Generator.

\subsubsection{AM Implementation}

Figure 6 shows AM implementation in system generator. In Figure 6(a), AM modulation system is considered. The carrier and the message signals are externally generated. Figure 6(b) illustrates the carrier signal and the message signal (discrete sine waves), in addition to the AM modulated signal. The used system generator blocks are: gateway in, gateway out, AddSub, Mult, constant, sine wave and the scopes.

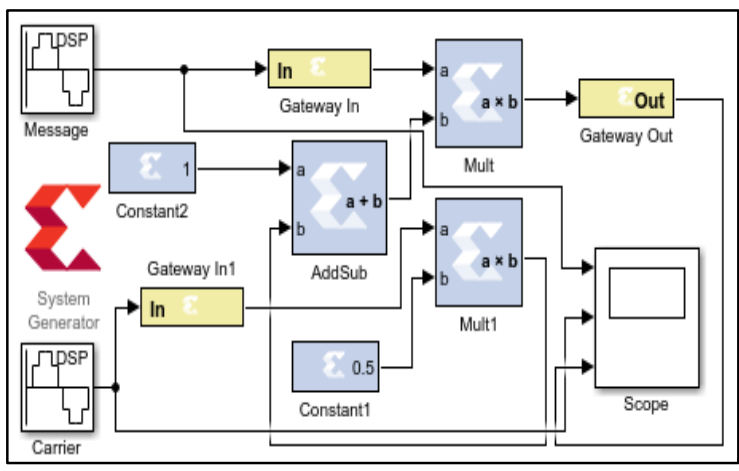

(a)

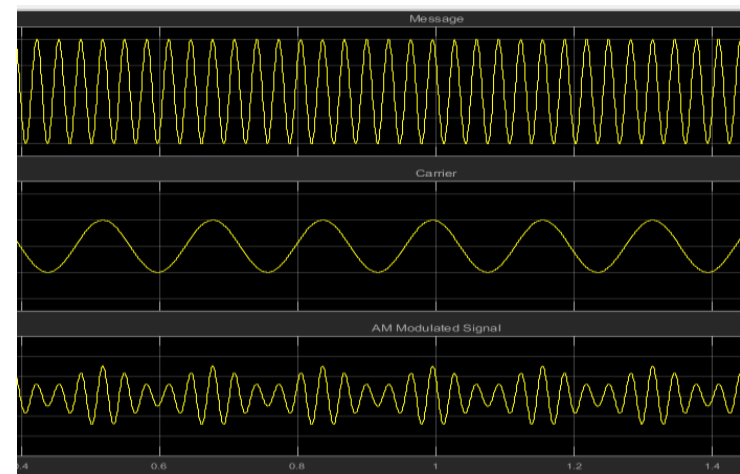

(b)

Figure 6. AM implementation in system generator. (a) AM Modulator in system generator, (b) The AM modulating and modulated signals

\subsubsection{FM Implementation}

Figure 7 shows FM implementation in system generator. In Figure 7(a), FM modulation system is considered. The message signal is externally generated. Figure 7(b) illustrates the message signal (discrete sine waves), in addition to the FM modulated signal. The used system generator blocks are: gateway in, gateway out, AddSub, Mult, constant, Accumulator, CMult, sine waves and the scopes. 


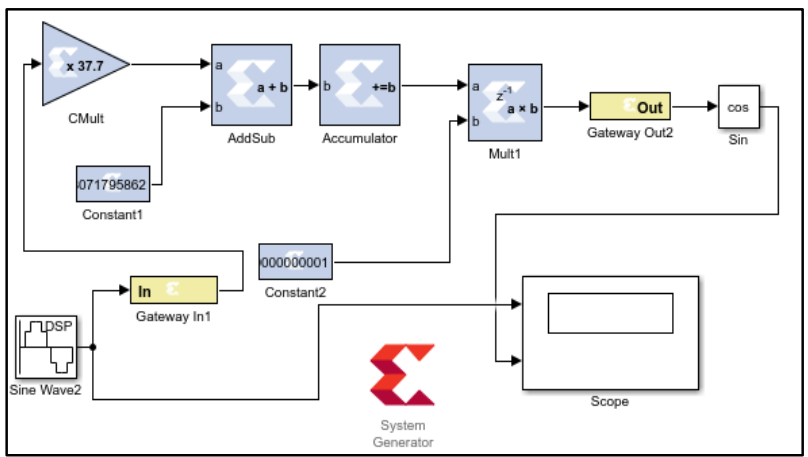

(a)

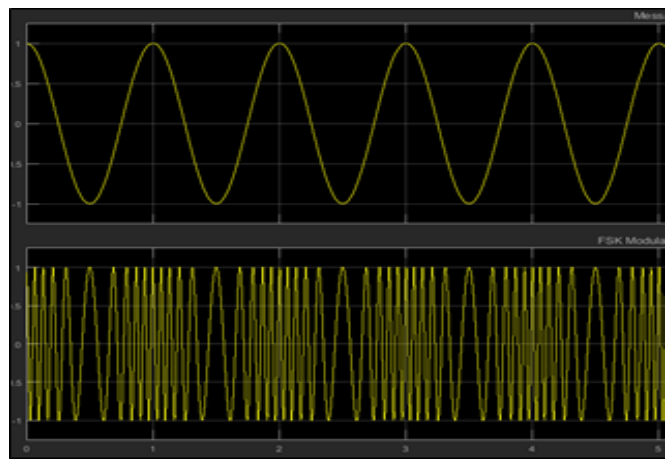

(b)

Figure 7. FM implementation in system generator. (a) FM Modulator in system generator,

(b) The FM modulating and modulated signals

\subsubsection{PM Implementation}

Figure 8 shows PM implementation in system generator. In Figure 8(a) PM system is considered, the message signal is externally generated. Figure 8(b) illustrates the message signal (discrete sine waves), in addition to the PM modulated signal. The used system generator blocks are: gateway in, gateway out, AddSub, Mult, constant blocks, accumulator blocks, CMult blocks, sine waves and the scopes.
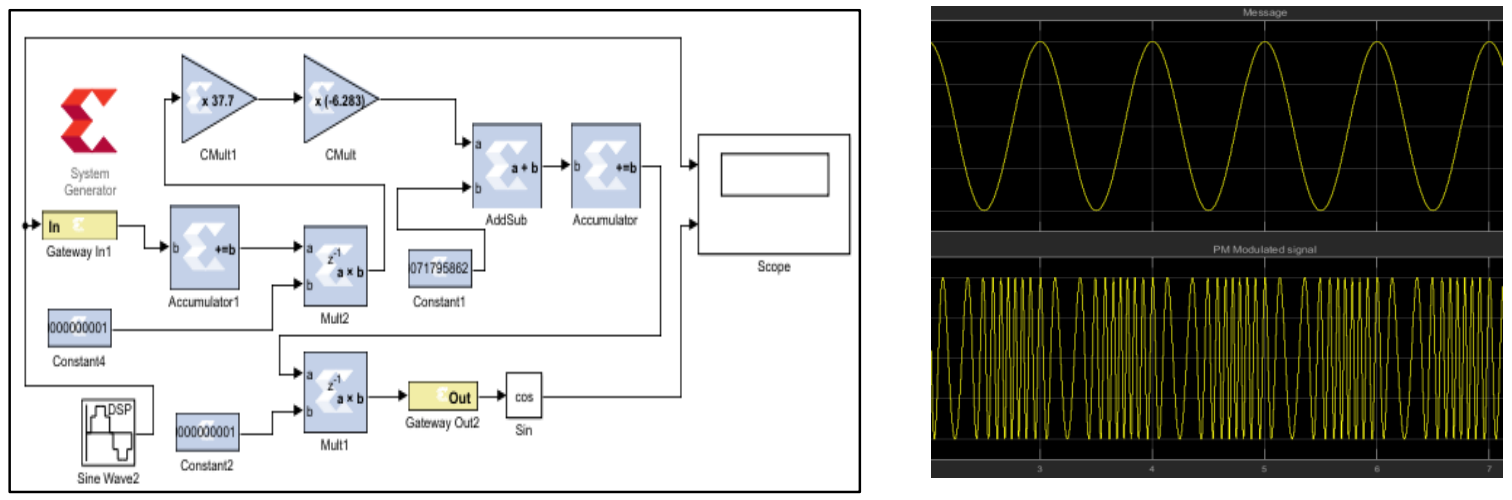

Figure 8. PM implementation in system generator. (a) PM Modulator in system generator,

(b) The PM modulating and modulated signals

\section{RESULTS AND DISCUSSION}

All the designed digital and analog modulation systems had been successfully converted to VHDL codes using HDL Netlist tools in system generator token with the appropriate settings of FPGA board and clock requirements. Then, VHDL codes are synthesized, implemented, and successfully converted to bitstreams that are downloadable to NEXYS4 DDR Board with ARTIX 7 XC7A100T-1CSG324C FPGA device. In system generator's environments, the input signals are demonstrated as external inputs in system generator, these inputs are successfully connected to FPGA board's input switches. The inputs and outputs to the gateway in and gateway out blocks are assigned successfully to the available input switches and output leds in the target board during the process of implementing the constrain file in Vivado. The process of programing the FPGA board with FSK bitstream is shown in Figure 9(b) Figure 9(a) show the schematic results of FSK modulation. Figure 10 shows FSK waveform simulation results. Table 1 shows the utilization summery of digital and analog modulation techniques such as: number of slice LUTs, Slice Register, Slice, LUT as Logic, LUT as memory, LUT flip flop pairs, and number of IO blocks. The table shows that the LUT as logic that used in FSK modulation is $0.13 \%$ while in FM modulation is $6.59 \%$. And also the Bounded IO that used in BPSK is $4.8 \%$ while in PM is $61.43 \%$. So that the parameters that used in analog implementation such as FM and PM are more than the parameters that used in digital modulation according to larger number of multipliers that required to implement the analog modulation. Only the parameter BUFGCTRL is the same in all implementation. 


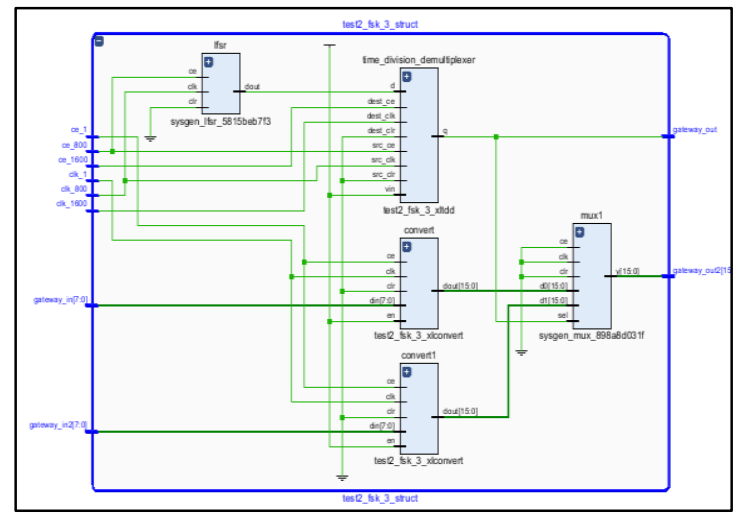

(a)

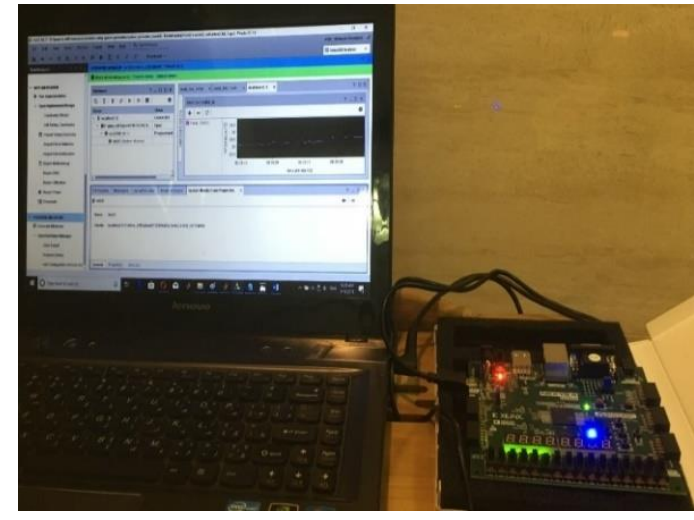

(b)

Figure 9. FSK hardware implementation. (a) Schematic results, (b) Programing FPGA board

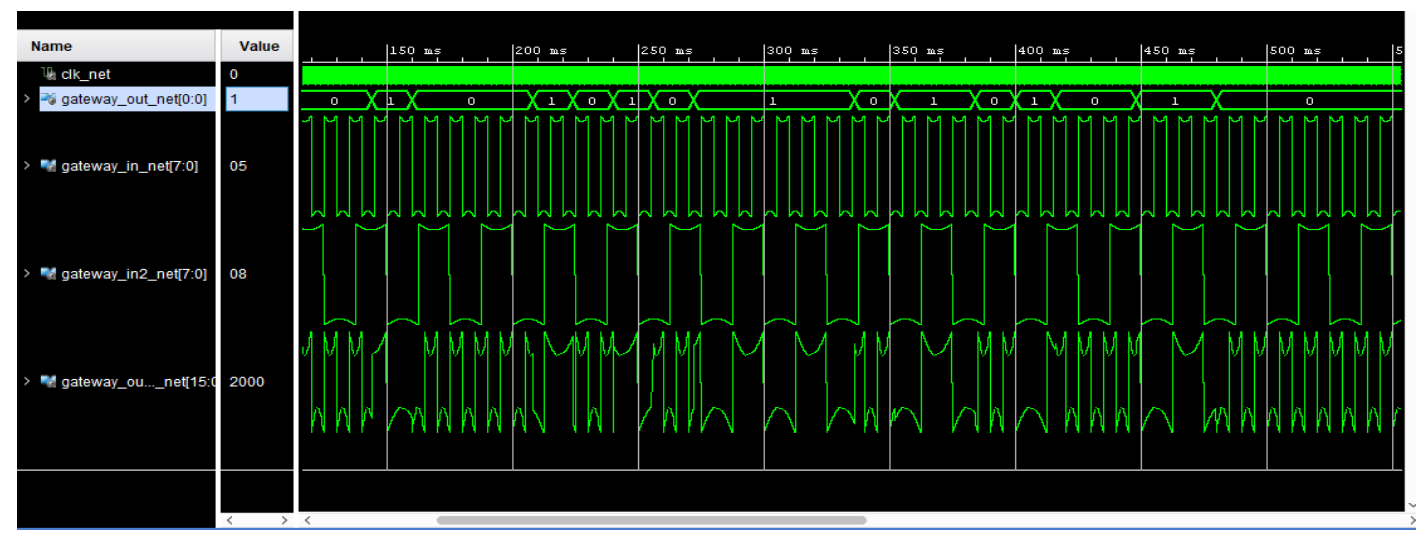

Figure 10. FSK simulation results

Table 1. Summery of Design Systems Utilization

\begin{tabular}{|c|c|c|c|c|c|c|c|}
\hline $\begin{array}{c}\text { Targeted device: } \\
\text { Xc7a100tcsg324-1 } \\
\text { (available) }\end{array}$ & $\begin{array}{c}\text { Slice LUTs } \\
63,400\end{array}$ & $\begin{array}{c}\text { Slice } \\
\text { Registers } \\
126800 \\
\end{array}$ & $\begin{array}{l}\text { Slice } \\
15850\end{array}$ & $\begin{array}{l}\text { LUT as } \\
\text { Logic } \\
63400 \\
\end{array}$ & $\begin{array}{c}\text { LUT flip } \\
\text { flop pairs } \\
63400 \\
\end{array}$ & $\begin{array}{c}\text { Bounded } \\
\text { IO } \\
210 \\
\end{array}$ & $\begin{array}{c}\text { BUFGCTRL } \\
32\end{array}$ \\
\hline BASK & $\begin{array}{c}49 \\
(0.07 \%)\end{array}$ & $\begin{array}{c}49 \\
(0.04 \%)\end{array}$ & $\begin{array}{c}20 \\
(0.126 \%)\end{array}$ & $\begin{array}{c}49 \\
(0.07 \%)\end{array}$ & $\begin{array}{c}23 \\
(0.04 \%)\end{array}$ & $\begin{array}{c}17 \\
(8 \%)\end{array}$ & $\begin{array}{c}1 \\
(3 \%)\end{array}$ \\
\hline BFSK & $\begin{array}{c}83 \\
(0.13 \%)\end{array}$ & $\begin{array}{c}78 \\
(0.06 \%)\end{array}$ & $\begin{array}{c}34 \\
(0.2 \%)\end{array}$ & $\begin{array}{c}83 \\
(0.13 \%)\end{array}$ & $\begin{array}{c}29 \\
(0.05 \%)\end{array}$ & $\begin{array}{c}10 \\
(4.8 \%)\end{array}$ & $\begin{array}{c}1 \\
(3 \%)\end{array}$ \\
\hline BPSK & $\begin{array}{c}77 \\
(0.12 \%)\end{array}$ & $\begin{array}{c}49 \\
(0.04 \%)\end{array}$ & $\begin{array}{c}23 \\
(0.15 \%)\end{array}$ & $\begin{array}{c}77 \\
(0.12 \%)\end{array}$ & $\begin{array}{c}23 \\
(0.04 \%)\end{array}$ & $\begin{array}{c}10 \\
(4.8 \%)\end{array}$ & $\begin{array}{c}1 \\
(3 \%)\end{array}$ \\
\hline DPSK & $\begin{array}{c}83 \\
(0.13 \%)\end{array}$ & $\begin{array}{c}82 \\
(0.06 \%)\end{array}$ & $\begin{array}{c}30 \\
(0.2 \%)\end{array}$ & $\begin{array}{c}82 \\
(0.13 \%)\end{array}$ & $\begin{array}{c}44 \\
(0.07 \%)\end{array}$ & $\begin{array}{c}18 \\
(9 \%)\end{array}$ & $\begin{array}{c}1 \\
(3 \%)\end{array}$ \\
\hline QPSK & $\begin{array}{c}47 \\
(0.07 \%)\end{array}$ & $\begin{array}{c}38 \\
(0.03 \%)\end{array}$ & $\begin{array}{c}21 \\
(0.13 \%)\end{array}$ & $\begin{array}{c}47 \\
(0.07 \%)\end{array}$ & $\begin{array}{c}15 \\
(0.02 \%)\end{array}$ & $\begin{array}{c}49 \\
(23 \%)\end{array}$ & $\begin{array}{c}1 \\
(3 \%)\end{array}$ \\
\hline $\mathrm{AM}$ & 0 & 0 & 0 & 0 & 0 & $\begin{array}{c}40 \\
(19.05 \%)\end{array}$ & $\begin{array}{c}0 \\
(0 \%)\end{array}$ \\
\hline FM & $\begin{array}{c}4177 \\
(6.59 \%)\end{array}$ & $\begin{array}{c}137 \\
(0.11 \%)\end{array}$ & $\begin{array}{c}1373 \\
(8.66 \%)\end{array}$ & $\begin{array}{c}4177 \\
(6.59 \%)\end{array}$ & $\begin{array}{c}60 \\
(0.09 \%)\end{array}$ & $\begin{array}{c}129 \\
(61.43 \%)\end{array}$ & $\begin{array}{c}1 \\
(3 \%)\end{array}$ \\
\hline PM & $\begin{array}{c}7644 \\
(12.06 \%)\end{array}$ & $\begin{array}{c}274 \\
(0.22 \%)\end{array}$ & $\begin{array}{c}2561 \\
(16.16 \%)\end{array}$ & $\begin{array}{c}7644 \\
(12.06 \%)\end{array}$ & $\begin{array}{c}122 \\
(0.19 \%)\end{array}$ & $\begin{array}{c}129 \\
(61.43 \%)\end{array}$ & $\begin{array}{c}1 \\
(3 \%)\end{array}$ \\
\hline
\end{tabular}

\section{CONCLUSION}

In this work, the design and implementation of analog and digital modulation techniques are obtained in system generator with Vivado 2017.4. The main contribution of this paper is the implementation of analog modulation in FPGA hardware. The FPGA implementation of digital modulation such as BASK, BFSK, BPSK, DPSK, and QPSK and analog modulation such as AM, FM and PM modulation techniques 
have been tested using NEXYS 4 ARTIX 7 DDR FPGA board. With the FPGA-based digital and analog modulation system, the results from this work proved that the numbers of FPGA resources required for digital modulation schemas are less than the ones required for analog modulation schemas.

\section{REFERENCES}

[1] S. Popescu, A. Gontean, G. Budura, "Hardware Co-Simulation of the BPSK and QPSK Systems on FPGA," IFAC Proceedings Volumes, vol. 45, no. 7, pp. 299-304, 2012.

[2] R. K. Mohammed, Y. UENO, "An FPGA-based Network Firewall with Expandable Rule Description," Indonesian Journal of Electrical Engineering and Computer Science, vol. 10, no. 3, pp. 1310-1318, 2018.

[3] H. A. Abdullah, H. N. Abdullah, "FPGA implementation of color image encryption using a new chaotic map," Indonesian Journal of Electrical Engineering and Computer Science, vol. 13, no. 1, pp. 129-137, 2019.

[4] Misbah, M. Rivai, F. Kurniawan, "Quartz crystal microbalance based electronic nose system implemented on Field Programmable Gate Array," TELKOMNIKA, vol. 17, no. 1, p. 370 376, 2019.

[5] G. Lema, T. Reda, D. Hailu, T. Sutikno, "Practical understanding of the operating principle of digital communication systems," Indonesian Journal of Electrical Engineering and Computer Science, vol. 16, no. 1, pp. 299 310, 2019.

[6] S. Rosmaniza AR, N. Ahmad, S. Syed Yusof, "Signal modulation techniques in non-orthogonal waveform for future wireless communication system," Indonesian Journal of Electrical Engineering and Computer Science, vol. 15 , no. 3 , p. $2019,1458 \sim 1465$.

[7] H. Lee, S. Shin, "A novel index modulation scheme with impedance matching," Indonesian Journal of Electrical Engineering and Computer Science, vol. 14, no. 3, p. 1203 1209, 2019.

[8] Suroso, D. Nugroho,A. Azis, T. Noguchi, "Simplified five-level voltage source inverter with level-phaseshifted carriers based modulation technique," Indonesian Journal of Electrical Engineering and Computer Science, vol. 3, no. 2, p. 461 468, 2019.

[9] P. Kolankar, S. Sakhare, "FPGA Implementation of QPSK modulator by using Hardware Co-simulation," International Journal of Engineering Research and Development, vol. 10, no. 4, pp. 86-93, 2014.

[10] J. Kamble, I. A. Pasha and M. Madhavilatha,, "FPGA Implementation of Digital Modulation Technique for HRR Target Detection," Indian Journal of Science and Technology, vol. 8, no. 24, p. 6, 2015.

[11] Bhaskara Rao Jammu, Hemanth Kumar Botcha,Nalini Bodasingi, "FPGA Implementation of BASK-BFSK-BPSKDPSK Digital Modulators using System Generator," in International Conference on circuits Power and Computing Technologies [ICCPCT], IEEE, Kollam, India, 2017.

[12] F. Quadri, A. D.Tete, "FPGA Implémentation of Digital Modulation Techniques," in International conference on Communication and Signal Processing, India, 2013.

[13] A. Sinha, P. Lotia, "A Study on FPGA Based Digital Modulators," International Journal of Advanced Research in Electrical,Electronics and Instrumentation Engineering, vol. 4, no. 4, pp. 1935-1942, 2015.

[14] P. N. MURTHY, "Design and Simulation of Reconfigurable Digital Modulator using Vivado System Generator," in Proceedings of the Third International Conference on Electronics Communication and Aerospace Technology, 2019.

[15] S. Samaddar, A. Sanyal, S. Sanyal, "FPGA based generalized architecture for Modulation and Demodulation Techniques," Journal of Computing Technologies, pp. 27-32, 2012.

[16] Hanan M. Hamee, Jafer Wadi, "Automatic Modulation Recognition for MFSK Using Modified Covariance Method," International Journal of Electrical and Computer Engineering (IJECE), vol. 5, no. 3, pp. 429-435, 2015.

[17] K. Mishra, S.K. Yadav, "Comparative Study of Digital Modulation Techniques Using MATLAB," International Journal of Innovative Research in Computer and Communication Engineering, vol. 3, no. 9, pp. 8486-8492, 2015.

[18] J. Boiko, V. Tolubko, O. Barabash, O. Eromenko, Y. Havrylko, "Signal processing with frequency and phase shift keying modulation in telecommunications," TELKOMNIKA, vol. 17, no. 4, p. 2025 2038, 2019.

[19] Q. Jun-suo, "A Algorithm of Fast Digital Phase Modulation Signal Recognition," TELKOMNIKA,, vol. 10, no. 8, pp. 2330-2335, 12.

[20] M. SÖNMEZ, A. AKBAL, "Design of a new BPSK modulator," Pamukkale University Journal of Engineering Sciences, vol. 23, no. 5, pp. 492-496, 2017.

[21] S. Choudhary, D. Bohra, "Study and simulation of digital modulation schemes using ber calculation," International Journal of Advance Research In Science And Engineering, vol. 4, no. 2, pp. 682-692, 2015.

[22] S. Jain,S. Yadav, "A Survey Paper on Digital Modulation Techniques," International Journal of Computer Sciences and Engineering, vol. 3, no. 12, pp. 107-111, 2015.

[23] R. Proloy, S. Ahmed, A. Hossain, "Comparative Analysis of Various Wireless Digital Modulation Techniques with different Channel Coding Schemes under AWGN Channel," International Journal of Computer Applications, vol. 161, no. 3, pp. 30-34, 2017.

[24] Vidyarani K R, Tejaswini S, Ankitha B M, Shalini D V, "Implementation of Analog Modulation Techniques using TMS320C6748 and LabVIEW," IOSR Journal of Research \& Method in Education (IOSR-JRME , vol. 7, no. 4, pp. 46-50, 2017.

[25] M. G. Ruppert, D. M. Harcombe, M. R. P. Ragazzon, S. O. Reza Moheimani, A. J. Fleming, "A review of demodulation techniques for amplitude-modulation atomic force microscopy," Beilstein Journal Nanotechnol, vol. 8, p. 1407-1426, 2017. 